This item was submitted to Loughborough's Research Repository by the author.

Items in Figshare are protected by copyright, with all rights reserved, unless otherwise indicated.

\title{
Fault diagnosis for vehicle lateral dynamics with robust threshold
}

PLEASE CITE THE PUBLISHED VERSION

http://dx.doi.org/10.1109/ICIT.2016.7475033

PUBLISHER

(c) IEEE

VERSION

AM (Accepted Manuscript)

LICENCE

CC BY-NC-ND 4.0

\section{REPOSITORY RECORD}

Su, Jinya, and Wen-Hua Chen. 2016. "Fault Diagnosis for Vehicle Lateral Dynamics with Robust Threshold". figshare. https://hdl.handle.net/2134/21198. 


\title{
Fault Diagnosis for Vehicle Lateral Dynamics with Robust Threshold
}

\author{
Jinya Su, Wen-Hua Chen \\ Department of Aeronautical and Automotive Engineering \\ Loughborough University, Loughborough LE11 3TU, U.K. \\ Email: J.Su2@lboro.ac.uk; W.Chen@lboro.ac.uk
}

\begin{abstract}
This paper investigates the robust fault diagnosis problem for vehicle lateral dynamics, which play a key role in vehicle stability and driving safety. The proposed fault diagnosis system consists of two sub-systems: fault diagnosis observer and robust threshold. By treating faults as disturbances, Disturbance/Uncertainty Estimation technique is used as fault diagnosis observer to generate residuals. Considering that residuals of model-based fault diagnosis are subject to the effect of uncertainties and consequently large false alarm rate may be resulted in, a novel robust threshold is then proposed based on reachability analysis technique for uncertain systems. The proposed fault diagnosis system is finally applied to the accelerometer and gyrometer sensor fault diagnosis problem of vehicle lateral dynamics, where initial states and velocity are considered to be uncertain. Simulation study verifies the effectiveness of the proposed fault diagnosis system.
\end{abstract}

Index Terms-Disturbance observer, Fault diagnosis, Reachability analysis, Robust threshold, Vehicle lateral dynamics.

\section{INTRODUCTION}

The use of automobiles is increasing worldwide, along with this trend are a significant number of accidents (every day about 3000 people die and 30000 people are seriously injured on the world's roads [1]). It was reported by United States DOT Report in 1992 that more than 90 percent of road accidents were caused by human errors [2]. Consequently, in the last few decades, much effort has been paid to developing advanced driver assistance systems (e.g., adaptive cruise control, lane keeping system, electronic stability control) to automate mundane driving operations such that drivers' burden and errors and consequently traffic accidents can thus be reduced.

To develop those advanced functions, new sensors, actuators and control units are inevitably introduced. For example, to improve active safety of vehicles due to loss of control, electronic stability control has been developed, which relies on a set of sensors (e.g., a gyrometer, an accelerometer, an encoder and a steering wheel sensor) and an upgraded integrated control unit [2]. In addition, to improve response time, safety and save weight, drive-by-wire systems have been developed, where conventional mechanical or hydraulic linkage has been gradually replaced by electronic or electromechanical systems. As a result, modern systems are becoming more complex and safety-critical, since the malfunction of components (e.g., sensors, actuators) may result in dangerous consequences [3].Vehicle lateral dynamics play a key role in vehicle maneuver-ability, stability and driving safety [2], [4], as a result, a lot of Fault Diagnosis (FD) algorithms have been proposed (see, [3]-[5]) to monitor actuator's or sensors' status.

Model-based FD, as a type of Analytical Redundancy (AR) approaches is receiving increasing attention recently [5]-[7]. Unlike Hardware Redundancy (HR) approach, they rely on mathematical models rather than redundant hardware and consequently do not result in additional weight and cost. They perform fault diagnosis by consistency-checking between observed behaviour and predicted behaviour using mathematical model of the concerned plant [6], [7]. There are generally two steps involved in this approach, including observer design to produce residuals (the residual is expected to be zero in the absence of faults and deviates from zero in the presence of faults) and threshold selection to evaluate residuals such that a Boolean decision can be made-normal or faulty. Different fault diagnosis observers are designed to generate residuals. Traditionally, output estimation errors (or their functions) are usually chosen as residuals [6], where a bank of observers are further designed for fault isolation. Recently, Disturbance/Uncertainty Estimation techniques are used as fault diagnosis observer by treating faults as disturbances, resulting in fault estimation based diagnosis approaches (see, [5], [7]-[9]). In this approach, fault estimates directly serve as residuals, which can substantially simplify fault isolation logic and reduce computation load.

However, no matter what kinds of fault diagnosis observers are designed, there is always an inevitable issue in modelbased fault diagnosis system, i.e., robustness. This is because residuals of fault diagnosis systems under normal case are not zero in real application, since there are always some mismatches between the real plant and the mathematical model used for observer design, such as system parameter uncertainties, external disturbances and sensor noises [10], [11]. For example, vehicle lateral dynamics depend on longitudinal velocity [3], [4], [12], which is uncertain due to measurement noises. Besides, it is also subject to bank angle, which can not be easily obtained and thus be treated as mismatched disturbances [13], [14]. As a result, many challenges are brought to the stage of robust threshold selection, since if the threshold is selected too small false alarm rate will be high and if selected too large missed detection rate will be high.

To solve the aforementioned challenging issue, this paper proposes a robust fault diagnosis system, where robustness 
is achieved in the stage of threshold selection. To this end, vehicle lateral dynamics are firstly described by systems with interval uncertainties [12]. Then a fault diagnosis observer (fault estimation based approach is chosen due to its simplicity in fault isolation) is designed based on the normal system such that residuals are generated. To achieve better robustness, a robust threshold selection approach is proposed based on reachability analysis, which can efficiently calculate all possible trajectories of an uncertain system in the presence of initial state uncertainty, parameter uncertainty and input uncertainty [15]. In this approach, all possible residual trajectories under normal case and a selected faulty cases are calculated in an over-approximation but tight way. Then a fault is alarmed when fault estimates deviate from residuals' normal reachable set. In this way, no false alarm will be present under described uncertainties, and users can also tune the missed detection rate through adjusting the uncertain intervals.

The rest of the paper is organized as follows. In Section. II, vehicle lateral dynamics are introduced. In Section. III, the proposed robust fault diagnosis system is discussed. Section. IV briefly introduces the reachability analysis tool, where examples are given to evaluate its effectiveness. The robust fault diagnosis system is applied to sensor fault diagnosis of vehicle lateral dynamic in Section. V. Section. VI concludes the paper and the future work is also discussed.

\section{VEHICLE LATERAL DYNAMIC}

In this section, vehicle lateral dynamics are introduced using one-track model, which is one of the most widely used models for the purpose of vehicle lateral control and fault diagnosis [2]-[5] due to its availability for on-line application and ability to well describe vehicle behaviour with lateral acceleration under $0.4 \mathrm{~g}$ ( $\mathrm{g}$ denotes acceleration of gravity) on normal dry asphalt roads [2]-[4]. The diagram of one-track model for vehicle lateral dynamics is shown in Fig. 1, where $\int r_{c}$ denotes the yaw angle and the rest notations are explained where needed.

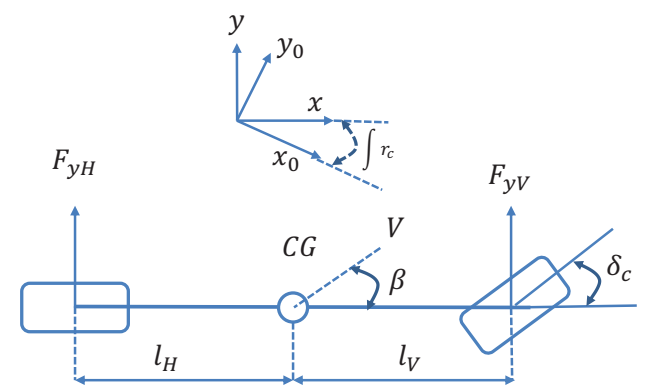

Fig. 1. The diagram of a one-track model for vehicle lateral dynamics.

Assuming a constant vehicle velocity (see, Chapter 2 of [2] or pp. 79 of [4] for other assumptions), taking vehicle side slip angle $\beta$ and yaw rate $r_{c}$ as state variables, and front wheel steering angle $\delta_{L}$ as input signal, vehicle lateral dynamics can be described by differential equations [2]

$$
\left\{\begin{array}{l}
m v\left(\dot{\beta}+\dot{r}_{c}\right)=F_{y V}+F_{y H} \\
I_{z} \dot{r}_{c}=l_{V} F_{y V}-l_{H} F_{y H}
\end{array}\right.
$$

where $m$ is vehicle mass, $v$ is vehicle longitudinal velocity, $F_{y V}, F_{y H}$ are front and rear tire forces, $I_{z}$ is yaw moment of inertia, and $l_{V}, l_{H}$ are distances from Centre of Gravity (CG) to front and rear tires, respectively.

Moreover, for small tire slip angles, lateral tire forces are usually approximated as a linear function of tire slip angles, which are defined

$$
\left\{\begin{array}{l}
F_{y V}=c_{\alpha V}\left(\delta_{c}-\beta-l_{V} r_{c} / v\right) \\
F_{y H}=c_{\alpha_{H}}\left(-\beta+l_{H} r_{c} / v\right)
\end{array}\right.
$$

where $c_{\alpha V}$ and $c_{\alpha H}$ are cornering stiffness of front and rear tires (values of all the parameters are given in Appendix).

Define vehicle state $x=\left[\beta ; r_{c}\right]$ and control input $u=\delta_{L}$, substituting (2) into (1), one can obtain the state-space model of vehicle lateral dynamic, given by

$$
\dot{x}=\left[\begin{array}{cc}
-\frac{c_{\alpha V}+c_{\alpha H}}{m v} & \frac{l_{H} c_{\alpha H}-l_{V} c_{\alpha V}}{m v^{2}}-1 \\
\frac{l_{H} c_{\alpha H}-l_{V} c_{\alpha V}}{I_{z}} & -\frac{l_{V}^{2} c_{\alpha V}+l_{H}^{2} c_{\alpha H}}{I_{z} v}
\end{array}\right] x+\left[\begin{array}{c}
\frac{c_{\alpha V}}{m v} \\
\frac{l_{V} c_{\alpha V}}{I_{z}}
\end{array}\right] u .
$$

The variables that can be directly measured are the lateral acceleration signal $\alpha_{y}$ through accelerometer and the yaw rate signal $r_{c}$ through gyrometer, i.e., $y=\left[\alpha_{y} ; r_{c}\right]$ and consequently the measurement model is

$$
y=\left[\begin{array}{cc}
-\frac{c_{\alpha V}+c_{\alpha H}}{m} & \frac{l_{H} c_{\alpha H}-l_{V} c_{\alpha V}}{m v} \\
0 & 1
\end{array}\right] x+\left[\begin{array}{c}
\frac{c_{\alpha V}}{m} \\
0
\end{array}\right] u .
$$

Since the velocity information is inevitably subject to measurement error, $v$ is treated as an uncertain parameter within a bounded interval [12]. Substituting the values of the rest of parameters [3], one can obtain the following compact form of vehicle lateral dynamic

$$
\begin{gathered}
\dot{x}=\underbrace{\left[\begin{array}{cc}
-\frac{85.449}{v} & \frac{28.774}{v^{2}}-1 \\
23.616 & -\frac{116.733}{v}
\end{array}\right]}_{A(v)} x+\underbrace{\left[\begin{array}{c}
\frac{35.236}{v} \\
33.258
\end{array}\right]}_{B(v)} u, \\
y=\underbrace{\left[\begin{array}{cc}
-85.450 & \frac{28.774}{v} \\
0 & 1
\end{array}\right]}_{C(v)} x+\underbrace{\left[\begin{array}{c}
35 \\
0
\end{array}\right]}_{D} u+F \underbrace{\left[\begin{array}{c}
f_{a} \\
f_{y}
\end{array}\right]}_{f} .
\end{gathered}
$$

In this model, the effect of sensor faults is also modelled through introducing fault variables (i.e., accelerometer fault $f_{a}$ and gyrometer fault $f_{y}$ ) and their distribution matrix $F$ (i.e., identity matrix). The under-brace notations $A(v), B(v), C(v)$ and $D$ are system matrix, input matrix, measurement matrix and feed-through matrix, respectively, where the first three matrices depend on uncertain velocity $v$.

The objective of fault diagnosis is to detect the presence of faults $f_{a}$ and $f_{y}$ (i.e., fault detection) and isolate which fault has occurred when a fault is detected (i.e., fault isolation). In this paper, model-based fault diagnosis approach is considered, 
which contains a fault diagnosis observer based on model (3) for residual generation and a robust threshold for residual evaluation. The diagram of the overall fault diagnosis system is shown in Fig. 2, where the fault diagnosis observer and robust threshold are discussed in Section. III-A and III-B respectively.

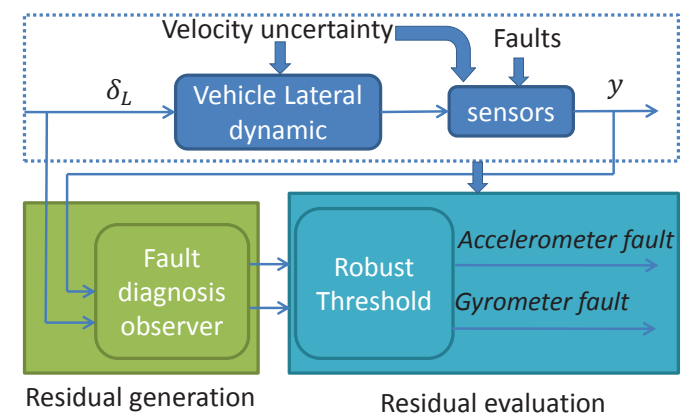

Fig. 2. The diagram of overall fault diagnosis system for vehicle latera dynamics including fault diagnosis observer and robust threshold selection.

\section{FAULT DiAGNOSIS SYSTEM}

In this section, the fault diagnosis system will be discussed, including fault diagnosis observer design to generate residuals and robust threshold selection to evaluate residuals.

When there is a small perturbation (or uncertainty) in velocity $v$, the matrix $A(v)$ (similarly $B(v)$ and $C(v)$ ) will fall into an interval matrix $\mathcal{A}$ (Matrices whose elements can take values within intervals are referred to as interval matrices), i.e., $A(v) \in \mathcal{A}$ where $\mathcal{A}=[\underline{A}, \bar{A}]$ is a interval matrix with $\underline{A}$ and $\bar{A}$ being the upper and low bound matrices element-wisely. The interval matrix $\mathcal{A}$ can also be equivalently represented by $\mathcal{A}=A+\Delta A$, where $A$ is the normal matrix and $\Delta A$ can be seen as the radius matrix element-wisely. As a result, the vehicle lateral dynamics with sensor faults (3) can be represented by the following generic systems

$$
\left\{\begin{array}{l}
\dot{x}=(A+\Delta A) x+(B+\Delta B) u \\
y=(C+\Delta C) x+D u+F f
\end{array} .\right.
$$

Consequently, in the following section, the fault diagnosis problem for generic system (4) will be considered.

\section{A. Fault diagnosis observer}

In this section, we consider fault diagnosis observer design for residual generation, whose role in the overall diagnosis system is shown in Fig. 2. The residual is chosen to indicate the presence of fault, and consequently it is expected to be close to zero in fault-free case and deviate from zero in the presence of faults [6]. In this paper, Disturbance/Uncertainty Estimation technique [5], [7], [9] is used as diagnosis observer by treating faults as disturbances, where the fault estimates serve as residuals for both fault detection and fault isolation.

Considering that the common sensor faults are bias, drift, scaling with unknown amplitude and occurring time, a linear model can effectively describe their dynamics [5]. Without loss of generality, the state augmentation approach is used for fault estimate, where a first-order model is chosen (see [5] for second-order models and [9] for high order models). In this approach, faults are augmented as additional states and can be estimated along with the original system states [7]. Taking $\bar{x}=(x, f)$, system (4) can be equivalently represented by the following extended system

$$
\left\{\begin{array}{l}
\dot{\bar{x}}=(\bar{A}+\Delta \bar{A}) \bar{x}+(\bar{B}+\Delta \bar{B}) u \\
y=(\bar{C}+\Delta \bar{C}) \bar{x}+D u
\end{array},\right.
$$

where $\bar{A}=\left[\begin{array}{cc}A & O \\ O & O\end{array}\right], \bar{B}=\left[\begin{array}{c}B \\ O\end{array}\right]$ and $\bar{C}=\left[\begin{array}{cc}C & F\end{array}\right]$.

Then a fault diagnosis observer can be designed for system (5) using normal matrices under the observability of the pair $(\bar{A}, \bar{C})$, as follows

$$
\left\{\begin{array}{l}
\dot{\bar{x}}=\bar{A} \hat{\bar{x}}+\bar{B} u+\bar{K}_{o}(y-\hat{y}) \\
\hat{y}=\bar{C} \hat{\bar{x}}+D u \\
\hat{f}=P \hat{\bar{x}}
\end{array},\right.
$$

where the gain matrix $\bar{K}_{o}$ is the observer gain matrix to be designed (e.g., pole assignment of the pair $(\bar{A}, \bar{C})$ ) and $P=$ $[O, I]$ is the projection matrix to obtain fault estimate from the augmented state estimate. Then fault estimates $\hat{f}$ serve as the residuals.

\section{B. Robust threshold}

The threshold is chosen to evaluate residuals such that false alarm rate and missed detection rate are kept at a low level. Our philosophy of robust threshold selection is as follows. We calculate the residual reachable set under normal case, i.e., in the presence of initial state uncertainties and system parameter uncertainties, then the upper and lower bounds of residual reachable set can be chosen as the robust threshold interval. And if fault estimates deviate from the threshold interval, a fault is alarmed. On the other hand, we can also calculate residual reachable set under a selected faulty cases. By doing so, we can determine the minimal fault amplitude that can be detected by a given fault diagnosis system.

However, the residual reachable set calculation for system (6) under normal case (i.e., in the presence of system uncertainties $\Delta \bar{A}, \Delta \bar{B}$ and $\Delta \bar{C}$ ) is never an easy task. This is because the input (i.e., $y$ ) to observer system (6) has infinite possible values at each time instant, since $y$ is the output of uncertain system (5). Infinite simulations are needed to cover the range of system parameter uncertainties and initial state uncertainties [16] and consequently the possible range of residuals. To this end, we transform this challenging issue into state reachable set calculation, as follows.

Combining (5) and (6), the error dynamic $e=\bar{x}-\hat{\bar{x}}$ can be obtained

$$
\dot{e}=\left(\bar{A}-\bar{K}_{o} \bar{C}\right) e+\left(\Delta \bar{A}-\bar{K}_{o} \Delta \bar{C}\right) \bar{x}+\Delta \bar{B} u .
$$

In addition, substituting measurement $y=(\bar{C}+\Delta \bar{C}) \bar{x}+D u$ of (5) into system (6), one can obtain

$$
\begin{aligned}
& \dot{\bar{x}}=\bar{A} \hat{\bar{x}}+\bar{B} u+\bar{K}_{o}(y-\hat{y}) \\
& =\bar{A} \hat{\bar{x}}+\bar{B} u+\bar{K}_{o}[(\bar{C}+\Delta \bar{C}) \bar{x}+D u-(\bar{C} \hat{\bar{x}}+D u)] \\
& =\bar{A} \hat{\bar{x}}+\bar{B} u+\bar{K}_{o}(\bar{C} e+\Delta \bar{C} \bar{x})
\end{aligned}
$$


Both system (7) and (8) involve unknown state variables $\bar{x}$, to make the residual reachability analysis feasible, we eliminate it through the relationship $\bar{x}=e+\hat{\bar{x}}$. Substituting $\bar{x}=e+\hat{\bar{x}}$ into (7) and (8) and putting them together, one can obtain

$$
\underbrace{\left[\begin{array}{c}
\dot{e} \\
\dot{\hat{x}}
\end{array}\right]}_{\dot{\chi}}=\underbrace{\left[\begin{array}{cc}
\bar{A}-\bar{K}_{o} \bar{C}+\Delta \bar{A}-\bar{K}_{o} \Delta \bar{C} & \Delta \bar{A}-\bar{K}_{o} \Delta \bar{C} \\
\bar{K}_{o} \bar{C}+\bar{K}_{o} \Delta \bar{C} & \bar{A}+\bar{K}_{o} \Delta \bar{C}
\end{array}\right]}_{\mathcal{A}} \underbrace{\left[\begin{array}{c}
e \\
\hat{x}
\end{array}\right]}_{\chi}+\underbrace{\left[\begin{array}{c}
\Delta \bar{B} \\
\bar{B}
\end{array}\right]}_{\mathcal{B}} u
$$

Using the under-brace notations in (9), Eq. (9) can be put into a compact form

$$
\dot{\chi}=\mathcal{A} \chi+\mathcal{B} u(t)
$$

Now the problem of residual reachable set calculation under normal and faulty cases has been transformed into the state reachability analysis of uncertain system (10) (since $\hat{f}$ is the elements of $\chi$ ), the implementation of which will be discussed in Section. IV.

\section{REACHABILITY ANALYSIS}

In this section, we briefly describe the tool of reachability analysis for uncertain system (10), where initial state uncertainties $x(0) \in X_{0}$, system parameter uncertainties $\mathcal{A}, \mathcal{B}$ and input uncertainties $u(t) \in U$ can be considered simultaneously. The exact set of reachable states $R_{e}^{d}(r)$ for a time $t=r$ is defined as:

$$
\begin{aligned}
R_{e}^{d}(r)= & \left\{x(r) \mid x(t)=\int_{0}^{t}[A x(\tau)+B u(\tau)] d \tau,\right. \\
& \left.x(0) \in X_{0}, A \in \mathcal{A}, B \in \mathcal{B}, \forall t: u(t) \in U\right\} .
\end{aligned}
$$

However, computation of exact reachable set for generic system is an open problem and consequently over-approximation is usually preferred [16].

1) Computation tool: The reachability analysis tool in [15], [16] is used in this paper, which can over-approximately (but in a tight way) obtain the reachable set $R([0, r])$ of system (10) in a time interval $t \in[0, r]$ denoted as $R^{d}([0, r]), R^{d}([0, r]) \supseteq$ $R_{e}^{d}([0, r])$ with $R_{e}^{d}([0, r])$ being the exact reachable set.

The detailed algorithm and its implementation can be found in [15], [16]. However, for the completeness of the paper, its basic algorithm structure for a time interval $\tau_{k}=\left[t_{k}, t_{k+1}\right]$ is given as follows. Partition $\mathcal{B} u(t)$ into $\mathcal{B} u(t)=u_{c}(t)+u_{u}(t)$ with $u_{c}(t)$ being the centre of uncertain input and $u_{u}(t)$ being $\mathcal{B} u(t)-u_{c}(t)$. Suppose the reachable set of the affine dynamics $\dot{x}=\mathcal{A} x+u_{c}(t)$ is $\mathcal{R}_{a}^{d}(t)$, the reachable set of the particular solution due to the uncertain input $u_{u}(t)$ is $\mathcal{R}_{p}^{d}\left(u_{u}(t), t\right)$, and the partial reachable set correcting the initial assumption that trajectories are straight lines between $t_{k}$ and $t_{k+1}$ is $\mathcal{R}_{\epsilon}^{d}$. Then the reachable set for a time interval $\tau_{k}$ is computed as shown in Fig. 3

i Starting from $\mathcal{R}^{d}\left(t_{k}\right)$, compute the reachable set $\mathcal{R}_{a}^{d}\left(t_{k+1}\right)$

ii Obtain the convex hull of $\mathcal{R}^{d}\left(t_{k}\right)$ and $\mathcal{R}^{d}\left(t_{k+1}\right)$ to approximate the reachable set for the time interval $\tau_{k}$

iii Compute $\mathcal{R}^{d}\left(\tau_{k}\right)$ by considering uncertain inputs by adding $\mathcal{R}_{p}^{d}\left(u_{u}(t), \tau_{k}\right)$ and accounting for the curvature of trajectories by adding $\mathcal{R}_{\epsilon}^{d}$.

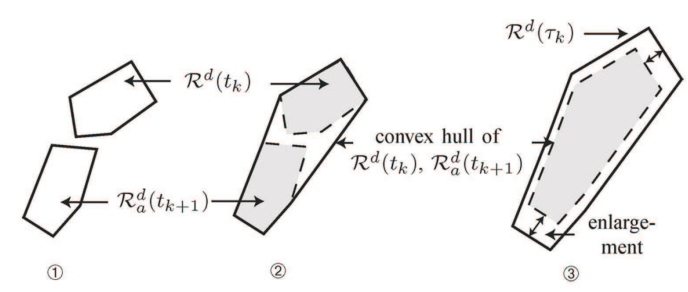

Fig. 3. Main steps for reachable set computation of an uncertain system.

The reachable set for the complete time interval is obtained by the union of the intermediate time interval through $R^{d}\left(\left[0, t_{f}\right]\right)=\bigcup_{k=1}^{t_{f} / r} R^{d}\left(\left[t_{k-1}, t_{k}\right]\right)$, where $r$ denotes the step.

2) Numerical verification: In this section, vehicle lateral dynamics (3) are used to evaluate the effectiveness of reachability analysis tool. From simulation results, the research challenges in threshold selection due to the presence of uncertainties will also be highlighted.

The vehicle velocity $v$ is assumed to be uncertain within a bounded interval $v \in[19,21] \mathrm{m} / \mathrm{sec}$, then following the procedure of [12], system matrices $A(v)$ and $B(v)$ in (3) can be calculated as interval matrices $A(v) \in A_{0}+[-1,1] \times$ $\Delta A, B(v) \in B_{0}+[-1,1] \times \Delta B$, with $A_{0}, \Delta A, B_{0}$ and $\Delta B$ given by

$$
\begin{gathered}
A_{0}=\left[\begin{array}{cc}
-4.2832 & -0.9275 \\
23.6162 & -5.8513
\end{array}\right], \Delta A=\left[\begin{array}{cc}
-0.2142 & -0.0073 \\
0 & -0.2926
\end{array}\right], \\
B_{0}=\left[\begin{array}{ll}
1.7662 & 33.2580
\end{array}\right], \Delta B=\left[\begin{array}{ll}
0.0883 & 0
\end{array}\right] .
\end{gathered}
$$

where $A_{0}$ is a stable matrix with complex poles.

The initial values of slip angle and yaw rate are within a bounded interval vector $([-0.02,0.02] ;[0.03,0.07])$. The known steering angle input $u_{c}(t)$ is a step input with amplitude $0.05 \mathrm{rad}$ at $1 \mathrm{sec}$. The step-size for reachability analysis is chosen as 0.01 and the order of zonotope $\rho$ is 400, under which configuration the computation time is less than $0.5 \mathrm{sec}$ using Matlab 2015a with Intel Core i5-3570 CUP @ 3.40 GHz. The reachable sets of slip angle $\beta$ and yaw rate $r_{c}$ together with 200 exemplary trajectories using stochastic Monte Carlo sampling are shown in Figs. 4 and 5 respectively.

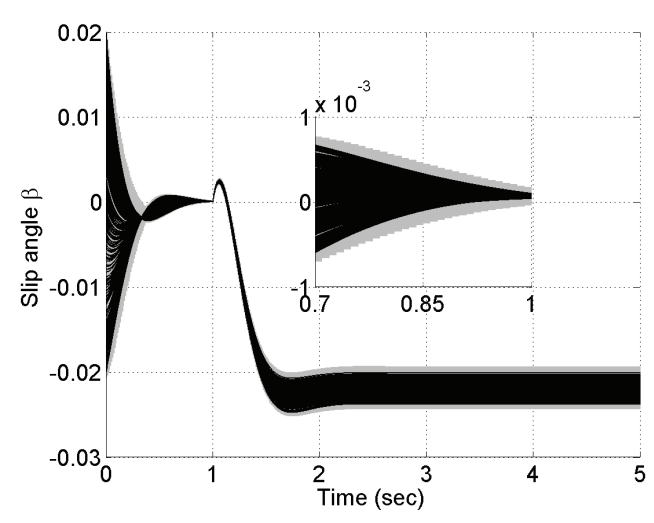

Fig. 4. The reachable set of slip angle (grey area) and 200 exemplary trajectories (black lines) with their zoom-in plot during 0.7 to $1 \mathrm{sec}$. 


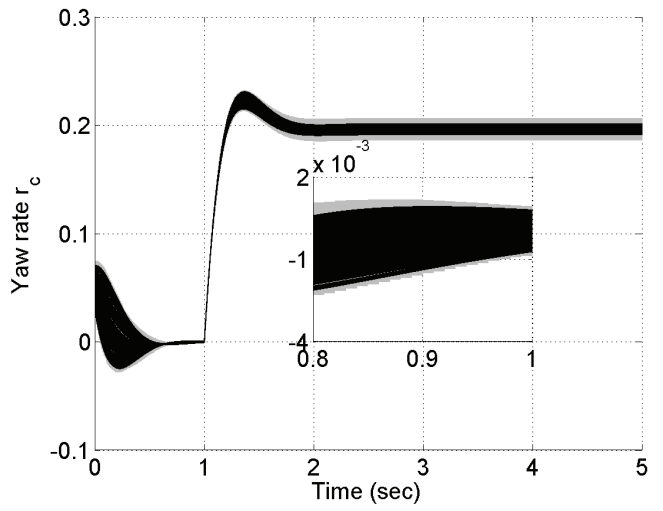

Fig. 5. The reachable set of yaw rate (grey area) and 200 exemplary trajectories (black lines) with their zoom-in plot during 0.8 to $1 \mathrm{sec}$.

We can see from Figs. 4 and 5 that: (i) all the exemplary trajectories lie in the calculated reachable sets, which verifies the effectiveness of reachability analysis tool; (ii) In the absence of control input, the reachable sets converge to zero due to system convergence; in the presence of control input after 1 sec, the reachable sets are no longer close to zero and the width of reachable sets are also increased compared with the case of without input (this is because the uncertainty $\Delta A x+\Delta B u$ in (4) becomes non-zero interval input). This phenomenon will bring many challenges to fault diagnosis, since in the presence of control input, the state reachable set and consequently the effect of system uncertainties on residuals will be obvious and can no longer be ignored.

\section{Application STUdy}

In this section, the robust fault diagnosis system in Section. III is applied to the sensor fault diagnosis problem of vehicle lateral dynamic systems described in Section. II. The accelerometer fault and gyrometer fault are considered simultaneously. The initial state of vehicle lateral dynamic systems is chosen as $x_{0}=[0 ; 0.05]$, and a steering input $u=0.05 \mathrm{rad}$ is executed at $1 \mathrm{sec}$. The accelerometer sensor fault and gyrometer sensor fault profile are plotted in red lines of Figs. 6 and 7.

The fault estimation based diagnosis observer is then designed using state augmentation approach, where accelerometer fault and gyrometer fault are treated as two additional states. The observer gain matrix $\bar{K}_{o}$ in (6) is designed using pole assignment of the normal system matrix pair $(\bar{A}, \bar{C})$ such that the poles of matrix $\bar{A}-\bar{K}_{o} \bar{C}$ are $[-10 ;-9 ;-4 ;-3]$. Since the initial system states can not be known exactly due to measurement errors, the initial states of the fault diagnosis observer are supposed to lie in a bounded interval, i.e., $\hat{\bar{x}}_{0}=[(-0.02,0.02),(0.03,0.07), 0,0]$, where uncertainties are supposed to exist in vehicle side slip angle and yaw rate.

The uncertain longitudinal velocity $v$ is supposed to lie in an uncertain interval $[19,21] \mathrm{m} / \mathrm{sec}$, based on which the uncertain matrices in Eq. (9) can be calculated using INTerval
LABoratory (INTLAB) as follows:

$$
\begin{gathered}
\Delta \bar{A}-\bar{K}_{o} \Delta \bar{C}=\left[\begin{array}{cccc}
-0.4283 & -0.0228 & 0 & 0 \\
0 & -0.5989 & 0 & 0 \\
0 & -0.5057 & 0 & 0 \\
0 & -0.0260 & 0 & 0
\end{array}\right], \\
\bar{K}_{o} \Delta \bar{C}=\left[\begin{array}{llll}
0 & 0.0042 & 0 & 0 \\
0 & 0.0069 & 0 & 0 \\
0 & 0.2529 & 0 & 0 \\
0 & 0.0130 & 0 & 0
\end{array}\right], \Delta \bar{B}=\left[\begin{array}{c}
0.0883 \\
0 \\
0 \\
0
\end{array}\right] .
\end{gathered}
$$

Based on them, the interval matrices $\mathcal{A}$ and $\mathcal{B}$ in (10) can be calculated. Now the initial state uncertainties, system matrix uncertainties in (10) are available, the reachability analysis tool described in Section. IV can be used to calculate the reachable sets of the accelerometer and gyrometer fault estimates (i.e., the residuals). The step-size for reachability analysis is chosen as 0.01 and the order of zonotope $\rho$ is 800 (the calculation accuracy can be further increased by reducing step-size and increasing zonotope order, which will require longer computation time), under which configuration the computation time is about 3 sec using Matlab 2015 with Intel Core i5-3570 CUP @ $3.40 \mathrm{GHz}$. The reachable sets of accelerometer and gyrometer fault estimates and exemplary trajectories using stochastic Monte Carlo Simulations are shown Figs. 6 and 7 respectively.

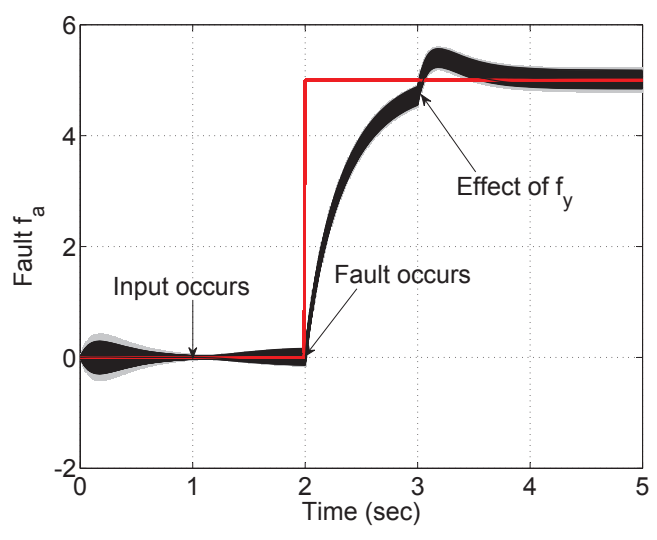

Fig. 6. Accelerometer fault profile (red line); accelerometer fault estimate reachable set (grey area) and stochastic simulated trajectories (black lines).

It can be observed in Figs. 6 and 7 that all the exemplary trajectories fall into the calculated reachable sets and the reachable sets are not overly conservative, i.e., the trajectories are not far away from the boundaries of the reachable sets, which again verifies the effectiveness of the reachability analysis tool.

During 0 to $1 \mathrm{sec}$, no steering control input is executed on the system, one can see the effect of initial state uncertainties and system parameter uncertainties on residuals, i.e., the residual reachable sets firstly get large and then converge close to zero. This is due to the fact that in the absence of control input, the system states of vehicle lateral dynamics and consequently the effect of parameter uncertainties will converge to zero in steady-state due to its convergence (i.e., the real parts of the eigenvalues of $A(v)$ are negative). After 


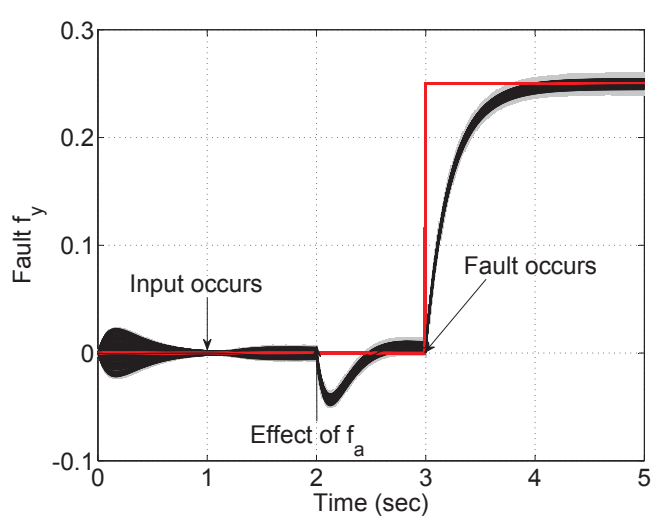

Fig. 7. Gyrometer fault profile (red line); gyrometer fault estimate reachable set (grey area) and stochastic simulated trajectories (black lines).

$1 \mathrm{sec}$, steering control input $u=0.05 \mathrm{rad}$ is given to the system, as a result, the system states of vehicle lateral dynamic systems and consequently the effect of system uncertainties on residuals will no long be zero (see also the simulation result in Section. IV).

We can see from Fig. 6, that in the presence of accelerometer fault at $2 \mathrm{sec}$, the accelerometer fault estimate reachable set substantially deviates from its normal reachable set before 2 sec, which verifies the effectiveness of fault diagnosis system. One can also see the effect of gyrometer fault on accelerometer fault reachable set at $3 \mathrm{sec}$ due to the coupling between them. Based on our robust threshold selection criteria, the boundaries of the reachable set of accelerometer fault estimate under normal case can be chosen as the interval threshold.

Similarly, we can see from Fig. 7 that in the presence of gyrometer sensor fault at $3 \mathrm{sec}$, the reachable set of gyrometer fault estimate substantially deviates from its normal reachable set. We can also see the effect of accelerometer fault on reachable set of gyrometer fault estimate at $2 \mathrm{sec}$. Similarly, the boundaries of the reachable set of gyrometer fault estimate under normal case can be chosen as the interval threshold.

\section{CONCLUSIONS AND FUtURE WORK}

In this paper, sensor fault diagnosis problem for vehicle lateral dynamics is considered using model-based fault diagnosis. The robust fault diagnosis system contains two subsystems including fault diagnosis observer and robust threshold selection. A fault estimation based diagnosis observer is first designed to produce residuals. A robust threshold is then proposed using reachability analysis to evaluate residuals such that the overall fault diagnosis system is robust to described system uncertainties and thus no false alarm appears.

In the future work, more realistic scenarios will be considered (e.g., other system parameter uncertainties such as cornering stiffness of front and rear tires, vehicle mass and sensor noise, etc.). Besides, the effect of bank angle can also be handled by enlarging the uncertain input interval. The results can also be extended to the threshold selection issue of the conventional residual based fault diagnosis approach.

\section{ACKNOWLEDGEMENT}

This work is supported by the UK Engineering and Physical Sciences Research Council (EPSRC) Autonomous and Intelligent Systems programme under the grant number $\mathrm{EP} / \mathrm{J} 011525 / 1$ with BAE Systems as the leading industrial partner.

\section{APPENDIX}

TABLE I

NUMERICAL VALUES OF THE PARAMETERS.

\begin{tabular}{|c|c|c|}
\hline Notation & Value & Significance \\
\hline $\mathrm{g}$ & $9.80 \mathrm{~m} / \mathrm{s}^{2}$ & gravity acceleration constant \\
\hline $\mathrm{m}$ & $1621 \mathrm{~kg}$ & vehicle total mass \\
\hline$l_{V}$ & $1.15 \mathrm{~m}$ & distance from CG to front axle \\
\hline$l_{H}$ & $1.38 \mathrm{~m}$ & distance from CG to rear axle \\
\hline$I_{z}$ & $1975 \mathrm{Kg} \cdot \mathbf{m}^{\mathbf{2}}$ & moment of inertia about the $\mathrm{z}$-axis \\
\hline$c_{\alpha V}$ & $57117 \mathrm{~N} / \mathrm{rad}$ & front axle tire cornering stiffness \\
\hline$c_{\alpha H}$ & $81396 \mathrm{~N} / \mathrm{rad}$ & rear axle tire cornering stiffness \\
\hline
\end{tabular}

\section{REFERENCES}

[1] C. Murray and A. D. Lopez, "Global health statistics: a compendium of incidence prevalence and mortality estimates for over 200 conditions.," 1996.

[2] R. Rajamani, Vehicle dynamics and control. Springer Science \& Business Media, 2011.

[3] S. Varrier, D. Koenig, and J. J. Martinez, "Robust fault detection for uncertain unknown inputs lpv system," Control Engineering Practice, vol. 22, pp. 125-134, 2014.

[4] M. El-ghatwary, Robust fuzzy observer-based fault detection for nonlinear systems. PhD thesis, Universität Duisburg-Essen, Fakultät für, 2007.

[5] Z. Gao, S. X. Ding, and Y. Ma, "Robust fault estimation approach and its application in vehicle lateral dynamic systems," Optimal Control Applications and Methods, vol. 28, no. 3, pp. 143-156, 2007.

[6] J. Chen and R. J. Patton, Robust model-based fault diagnosis for dynamic systems, vol. 3. Springer Science \& Business Media, 2012.

[7] J. Su, W.-H. Chen, and B. Li, "Disturbance observer based fault diagnosis," in Control Conference (CCC), 2014 33rd Chinese, pp. 30243029, IEEE, 2014.

[8] B. Jiang and F. N. Chowdhury, "Fault estimation and accommodation for linear mimo discrete-time systems," Control Systems Technology, IEEE Transactions on, vol. 13, no. 3, pp. 493-499, 2005.

[9] J. Su, W.-H. Chen, and B. Li, "High order disturbance observer design for linear and nonlinear systems," in International Conference on Information and Automation, IEEE, 2015, available on line.

[10] J. Su, B. Li, and W.-H. Chen, "On existence, optimality and asymptotic stability of the kalman filter with partially observed inputs," Automatica, vol. 53, pp. 149-154, 2015.

[11] J. Su, J. Yang, and S. Li, "Continuous finite-time anti-disturbance control for a class of uncertain nonlinear system," Transactions of the Institute of Measurement and Control, vol. 36, no. 3, pp. 300-311, 2014.

[12] M. Althoff, D. Althoff, D. Wollherr, and M. Buss, "Safety verification of autonomous vehicles for coordinated evasive maneuvers," in Intelligent vehicles symposium (IV), 2010 IEEE, pp. 1078-1083, IEEE, 2010.

[13] J. Yang, S. Li, J. Su, and X. Yu, "Continuous nonsingular terminal sliding mode control for systems with mismatched disturbances," Automatica, vol. 49, no. 7, pp. 2287-2291, 2013.

[14] J. Yang, J. Su, S. Li, and X. Yu, "High-order mismatched disturbance compensation for motion control systems via a continuous dynamic sliding-mode approach," Industrial Informatics, IEEE Transactions on, vol. 10, no. 1, pp. 604-614, 2014.

[15] M. Althoff, O. Stursberg, and M. Buss, "Reachability analysis of linear systems with uncertain parameters and inputs," in Decision and Control, 2007 46th IEEE Conference on, pp. 726-732, IEEE, 2007.

[16] M. Althoff and J. M. Dolan, "Online verification of automated road vehicles using reachability analysis," Robotics, IEEE Transactions on, vol. 30, no. 4, pp. 903-918, 2014. 